\title{
Phase II Study of Preoperative Radiation with Concurrent Capecitabine, Oxaliplatin and Bevacizumab Followed by Surgery and Postoperative 5-FU, Leucovorin, Oxaliplatin (FOLFOX) and Bevacizumab in Patients with Locally Advanced Rectal Cancer: ECOG 3204
}

\author{
Jerome C. Landry, M.D. ${ }^{1}$, Yang Feng, M.P.H., M.S. ${ }^{2}$, Steven J. Cohen, M.D. ${ }^{3}$, Charles A. \\ Staley III, M.D. ${ }^{1}$, Richard Whittington, M.D. ${ }^{4}$, Elin Ruth Sigurdson, M.D. ${ }^{3}$, Halla Nimeiri, M.D. \\ ${ }^{5}$, Udit Verma, M.D. ${ }^{6}$, Roshan S. Prabhu, M.D. ${ }^{1}$, and Al Bowen Benson, M.D. ${ }^{5}$ \\ ${ }^{1}$ Emory University and Winship Cancer Institute, Atlanta, Georgia \\ 2 Dana Farber Cancer Institute, Boston, Massachusetts \\ ${ }^{3}$ Fox Chase Cancer Center, Philadelphia, Pennsylvania \\ 4 Philadelphia VA Medical Center, Philadelphia, Pennsylvania \\ ${ }^{5}$ Northwestern University, Chicago, Illinois \\ ${ }^{6}$ University of Texas Southwestern Medical Center, Dallas, Texas
}

\section{Abstract}

\begin{abstract}
Background-Recent studies have demonstrated the feasibility of combining oxaliplatin with 5FU or capecitibine and radiation therapy (RT). The addition of bevacizumab to chemotherapy improves overall survival for metastatic disease. We initiated a phase II trial to evaluate preoperative capecitabine, oxaliplatin, and bevacizumab with RT followed by surgery and postoperative 5-FU, leucovorin, oxaliplatin (FOLFOX) and bevacizumab for locally advanced rectal cancer.
\end{abstract}

\begin{abstract}
Methods-Fifty-seven patients (pts) with resectable T3/T4 rectal adenocarcinoma were enrolled. Preoperative treatment was capecitabine $\left(825 \mathrm{mg} / \mathrm{m}^{2}\right.$ bid M-F), oxaliplatin $\left(50 \mathrm{mg} / \mathrm{m}^{2}\right.$ weekly), bevacizumab $(5 \mathrm{mg} / \mathrm{kg} \mathrm{D1}, 15,29)$, and RT $(50.4 \mathrm{~Gy})$. Surgery was performed by 6 weeks after neoadjuvant therapy. Beginning $8-12$ weeks after surgery, patients received FOLFOX plus bevacizumab $(5 \mathrm{mg} / \mathrm{kg})$ Q2 weeks for 12 cycles.
\end{abstract}

Results-Fifty-four of 57 enrolled pts were eligible. Forty-nine (91\%) pts completed preoperative therapy and underwent surgery. Nine pts (17\%, 90\% CI: [9\%-27\%]) achieved pathologic complete response (path CR). Thirty-two pts (59\%) experienced pathologic tumor downstaging. Fifty-three percent and 15\% of pts experienced worst grade 3 and grade 4 acute

Corresponding author: Jerome C. Landry, MD Professor Department of Radiation Oncology Emory University 1365 Clifton Road NE Atlanta, GA 30322 Phone: 404-778-3473 Fax: 404-778-4139 jland01@emory.edu.

This manuscript has not been previously published and is not under consideration in the same or substantially similar form in any other peer-reviewed media.

All authors have read and approved the manuscript. To the best of our knowledge, no conflict of interest, financial or other, exists except:

Steven J Cohen: honoraria from Genentech, Inc.

Halla Nimeiri: honoraria from Genentech, Inc.

Al Bowen Benson: consultant/advisor for Sanofi and Genentech, Inc. Research funding from Genentech, Inc. 
toxicity, respectively. Forty-seven percent of pts who underwent surgery experienced a surgical complication.

Conclusions-The primary endpoint of a $30 \%$ path CR rate was not reached, however the majority of patients experienced pathologic downstaging with this regimen. Increased wound healing delays and complications may have been related to the addition of bevacizumab, oxaliplatin, or both. Continued observation of these patients will establish the long term morbidity and efficacy of this combined modality approach.

\section{Keywords}

Rectal Cancer; Colorectal Cancer; Radiation therapy; Chemoradiation

\section{INTRODUCTION}

Colorectal cancer is the third most commonly diagnosed cancer in the United States and the third leading cause of death in males and females ${ }^{1}$. Approximately $28 \%$ of colorectal cancers arise in the rectum, accounting for 39,870 new cases of rectal cancer diagnosed in the United States in $2011^{1}$. Chemoradiation (CRT) with continuous infusion fluorouracil (CI 5-FU) in addition to surgery is recommended for locally advanced rectal cancer (stage II - III) due to excessive local recurrence rates with surgical resection alone ${ }^{2,3}$. Neoadjuvant 5-FU-based CRT has become the standard of care for stage II - III rectal cancer in the United States due to the findings of improved local control, higher rates of sphincter sparing procedures, and reduced toxicity versus adjuvant CRT from the German Rectal Cancer Study Group phase III trial ${ }^{4}$.

Capecitabine (Genentech Inc., San Francisco, CA) is an oral fluoropyrimidine which was rationally designed as a prodrug to deliver 5-FU predominately to the tumor tissue. It is rapidly and extensively absorbed as an intact molecule and is then metabolized to 5-FU. Oral capecitabine has been shown to be as effective as CI 5-FU for the treatment of locally advanced rectal cancer when given concurrently with neoadjuvant radiation therapy (RT) ${ }^{5}$. Oxaliplatin, a third-generation platinum derivative, is the only platinum agent with clinical activity in colorectal cancer, both directly and as a radiosensitizer ${ }^{6}$; it also has significant additive or synergistic activity with 5-FU ${ }^{7}$. At the time of initiation of this trial, the clinical efficacy of the addition of oxaliplatin to neoadjuvant fluoropyrimidine-based CRT was not yet known. Preliminary results from several phase III randomized trials have since demonstrated higher toxicity rates without benefit in terms of pathologic complete response (path CR) rate with the addition of oxaliplatin to standard neoadjuvant CRT ${ }^{5,8}$.

Bevacizumab (Genentech Inc., San Francisco, CA) is a recombinant humanized monoclonal antibody that acts against circulating vascular endothelial growth factor (VEGF). The addition of bevacizumab to chemotherapy for metastatic colorectal cancer resulted in a significant $34 \%$ relative reduction in risk of death versus chemotherapy alone ${ }^{9}$. Bevacizumab has also been shown to have synergistic anti-tumor effects with RT in preclinical models 10,11 . Several small studies have demonstrated the feasibility of integrating bevacizumab into fluoropyrimidine-based neoadjuvant CRT, with path CR rates ranging from $8 \%$ to $32 \% 12-14$.

Based on these findings, we initiated a multicenter phase II trial of preoperative capecitabine, oxaliplatin, and bevacizumab with RT followed by surgery and postoperative 5-FU, leucovorin, oxaliplatin (FOLFOX) and bevacizumab for resectable locally advanced rectal cancer. The purpose of this trial was to evaluate the safety, tolerability, and efficacy of this regimen with a primary endpoint of pathologic complete response. 


\section{PATIENTS AND METHODS}

\section{Eligibility Criteria}

Patients $\geq 18$ years old with histologically confirmed, non-metastatic, primary T3 or T4 adenocarcinoma of the rectum within $12 \mathrm{~cm}$ of the anal verge by proctoscopic examination were eligible for the study. Patients were staged according to the AJCC $6^{\text {th }}$ edition staging system $^{3}$. Additional eligibility criteria included T3/T4 staging by CT scan plus endorectal ultrasound (EUS) or a MRI, tumor prospectively defined by surgeon as either initially resectable or potentially resectable after preoperative chemoradiation, good general condition allowing major surgery (Eastern Cooperative Oncology Group performance status $0-1)$, normal liver, renal, and bone marrow function, and a written informed consent. Exclusion criteria included: tumor outside of the true pelvis, high-grade large bowel obstruction (lumen diameter $<1 \mathrm{~cm}$ ), unless diverting colostomy has been performed, pregnant or breast feeding female patient, prior chemotherapy for rectal cancer or any prior pelvic irradiation, history of stroke or transient ischemic attack at any time, myocardial infarction/unstable angina within 12 months of study entry, or any other serious condition or comorbidity that could potentially interfere with administration of trial therapy or follow-up. Patients with clinically significant congestive heart failure, peripheral vascular disease, bleeding diathesis, uncontrolled hypertension $\geq 150 / 100$ or $>$ grade 1 neuropathy were also excluded. Patients must have been disease-free from any other malignancy for $\geq 5$ years, with the exception of non-melanomatous skin cancer or cervical carcinoma in situ. The trial was approved by the local institutional review boards of all participating centers. This trial was registered with clinicaltrials.gov (identifier NCT00321685).

\section{Interventions}

Pre-operative chemotherapy-Preoperative chemotherapy consisted of capecitabine ( $825 \mathrm{mg} / \mathrm{m}^{2}$ q12 hours, by mouth, 5 days per week during RT), oxaliplatin $\left(50 \mathrm{mg} / \mathrm{m}^{2}\right.$, IV over 2 hours, once per week, days 1, 8, 15, 22, 29 of RT), and bevacizumab $(5 \mathrm{mg} / \mathrm{kg}$, IV over 30 - 90 minutes, once every other week, days 1,15, 29 of RT).

Radiation therapy-Standard or conformal treatment planning was allowed. Intensity modulated radiation therapy (IMRT) was not allowed. Linear accelerator based treatment with a minimum photon energy of $4 \mathrm{MV}$ was required. All patients underwent simulation with oral contrast to visualize the small bowel. The gross tumor volume (GTV) was defined as the rectum, perirectal and internal iliac lymph nodes, and the full extent of the presurgical mass. The clinical target volume (CTV) was defined as the GTV with a $2 \mathrm{~cm}$ margin, additionally including the pre-sacral space. The initial planning target volume (PTV1) was defined as the CTV with a minimum of $1 \mathrm{~cm}$ margin. Beam arrangements could be 3 field (posterior with opposed lateral fields) or 4 field (anterior/posterior and opposed lateral fields). $45 \mathrm{~Gy}$ in 25 fractions of $1.8 \mathrm{~Gy}$ per day, 5 days per week, over 5 weeks, was delivered to the PTV1. An additional boost was then delivered to the boost PTV, which was defined as the primary tumor and lymph nodes $>1.5 \mathrm{~cm}$ with a $2 \mathrm{~cm}$ margin. The boost was 5.4 Gy over 3 fractions, for a total of 50.4 Gy given at 1.8 Gy per fraction for 28 fractions, over 5.5 weeks.

Quality assurance for RT consisted of review of the treatment fields, pre-study diagnostic imaging, dose-volume histograms, dose distributions, and portal imaging by the Quality Assurance Review Center (QARC) within 3 days of the start of RT. All linear accelerator units used in this study had their calibration verified by the Radiological Physics Center (RPC). 
Surgery-Surgery was scheduled to occur $6-8$ weeks after the completion of preoperative CRT. Patients were locally restaged using the same imaging modality that was used pre-study (CT and EUS or MRI). Restaging prior to surgery also included a chest x-ray or CT of the chest. The choice of operative procedure was at the discretion of the treating surgeon. It was strongly recommended that the entire mesorectum be removed and that a distal rectal margin of $\geq 2 \mathrm{~cm}$ be obtained for sphincter preserving procedures.

Post-operative chemotherapy-Postoperative chemotherapy was administered to all patients who had a macroscopic complete resection, beginning 4 - 12 weeks after surgery. Post-operative chemotherapy consisted of leucovorin ( $400 \mathrm{mg} / \mathrm{m}^{2}$, IV over 2 hours, day 1), 5 -FU ( $400 \mathrm{mg} / \mathrm{m}^{2}$, IV bolus, day 1$), 5$-FU $\left(2400 \mathrm{mg} / \mathrm{m}^{2}\right.$, continuous IV infusion over 46 hours, days 1-2), oxaliplatin ( $85 \mathrm{mg} / \mathrm{m}^{2}$, IV infusion over 2 hours, day 1$)$, and bevacizumab $(5 \mathrm{mg} / \mathrm{kg}$, IV infusion over 90 minutes, day 1). Cycles were repeated every 2 weeks for a total of 12 (2 week) cycles, except for oxaliplatin, which was administered for 9 cycles only.

\section{Follow-Up}

Toxicity was graded according to the National Cancer Institute's Common Terminology Criteria for Adverse Events (version 3.0 through 6/30/2011, version 4.0 after 6/30/2011). Patients were assessed weekly during neoadjuvant CRT, prior to surgical resection, and prior to each adjuvant chemotherapy cycle. After completion of all therapy, patients were assessed every 3 months for the first 2 years. Afterwards, follow-up was planned for every 6 months up to 7 years, and every year thereafter. Pathologic response was assessed in all patients who underwent surgical resection. A complete pathologic response (path CR) was defined as no evidence of malignancy in the primary or nodal surgical specimens. Acute preoperative toxicity was defined as adverse events that occurred during neoadjuvant chemoradiation or after neoadjuvant therapy, but prior to surgery. Post-surgical complications were defined as adverse events that occurred during the inpatient hospital stay in the immediate post-operative period. Late surgical complications were defined as adverse events that occurred after the initial post-surgical hospital discharge.

\section{Statistical Considerations}

The primary endpoint of this study was pathologic complete response. Fifty-five eligible patients were planned to be accrued in this trial. To allow for 5\% ineligibility, 58 patients were planned to be entered. If the treatment was indicative of a true path CR rate of $30 \%$, we would consider it a promising regimen for further study. A true path CR rate of less than $15 \%$ was considered unpromising. To limit accrual if the treatment was not effective, a twostage design was used, allowing early stopping if the true path CR rate was less than $15 \%$. If at least 4 pathologic complete responses were observed among the first 23 eligible patients, 34 additional patients (assuming 32 eligible) would be entered in the second stage. Five of the first 15 patients (33\%) who completed preoperative therapy and surgery demonstrated a path CR after the first stage of accrual, and the decision was made to continue to the second stage ${ }^{15}$. If 12 or more pathologic complete responses are seen in the 55 eligible patients, the treatment will be considered promising.

\section{RESULTS}

\section{Patient Characteristics}

A total of 57 patients were enrolled between 07/2006 and 05/2010 from 11 participating sites. Of the 57 patients, 1 was found ineligible due to T2 disease and 2 patients never started treatment, leaving 54 eligible patients that were included in the analysis. Data updated through 11/8/2011 were analyzed. At the time of analysis, 4 of the 54 eligible patients had data collected that had not been centrally reviewed. The median follow-up 
period for alive patients was 17 months (range $2-54$ ). The median age was 54 years (range 26 - 83). Clinical T stage was T3 for 50 patients (93\%) and T4 for 4 patients (7\%). Clinical nodal stage was NX for 2 patients (4\%), N0 for $16(30 \%)$, N1 for $31(57 \%)$, and N2 for 5 $(9 \%)$. Table 1 summarizes patient demographics.

\section{Treatment Characteristics}

Forty-nine patients (91\%) completed preoperative CRT, all of whom underwent curative surgical resection. Forty-four patients (82\%) completed preoperative CRT per protocol or with minor deviations only. The median radiation dose delivered was $50.4 \mathrm{~Gy}$ (range $50-$ $50.4 \mathrm{~Gy}$ ). The median time period from the last chemotherapy cycle to surgery was 7.6 weeks (range 5.3 - 11.9 weeks), and the median time period to initiation of adjuvant chemotherapy after surgery was 9 weeks (range $6-14$ weeks). Sixty-seven percent of patients underwent surgical resection within 8 weeks of completion of neoadjuvant CRT. Five patients (9\%) did not undergo surgical resection. Reasons for not undergoing going surgery were patient refusal $(n=1)$, adverse event $(n=2)$, and patient death during neoadjuvant CRT $(n=2)$. Of the 49 patients who underwent curative surgery, $25(51 \%)$ began adjuvant chemotherapy. Reason for not starting adjuvant chemotherapy included wound complication for 13 patients and patient refusal for 8 patients. For the 25 patients who began adjuvant chemotherapy, the median number of cycles completed was 12 (range 1 -12). Sixteen patients (30\%) received all 12 cycles and completed the entire treatment per protocol.

\section{Preoperative Chemoradiation Toxicity}

Fifty-five patients were included in the toxicity analysis: 54 eligible patients and 1 patient with a T2 tumor who underwent study therapy, but was later found to be ineligible. Two patients (4\%) experienced grade 5 events during preoperative CRT, one of which was secondary to aspiration and attributed to study therapy. The other patient died during the third week of neoadjuvant CRT that was reported as not associated with study therapy and definitely related to rectal cancer. Overall, there were 29 patients (53\%) with worst grade 3 toxicity and 8 patients (15\%) with worst grade 4 toxicity during preoperative therapy. A total $16(29 \%)$ and 4 (7\%) patients experienced grade 3 and grade 4 hematologic toxicity, respectively. The most common hematologic grade $3 / 4$ toxicities were neutropenia (18\%), leukopenia (15\%), and lymphopenia (15\%). Table 2 summarizes hematologic toxicity. A total of $25(45 \%)$ and $7(13 \%)$ patients experienced grade 3 and grade 4 non-hematologic toxicity, respectively. The most common nonhematologic grade $3 / 4$ toxicities were diarrhea $(13 \%)$, fatigue (15\%), and rectal pain (16\%). Table 3 summarizes the most common nonhematologic toxicity.

\section{Postoperative Complications}

The median time interval from last bevacizumab administration to surgery was 7.6 weeks (range 5.3 - 11.9 weeks). Forty-nine patients underwent surgical resection and were included in the post-surgical toxicity analysis. A total of 9 patients (18\%) experienced post-surgical complications, including wound infection $(n=8)$, fascial dehiscence $(n=5)$, intraabdomominal abscess $(\mathrm{n}=1)$, fistula $(\mathrm{n}=1)$, bowel obstruction $(\mathrm{n}=1)$, and thrombosis/ embolism $(n=1) .3$ patients $(6 \%)$ required surgical intervention for post-surgical complications. These consisted of intra-abdominal abscess drainage (1 patient), perirectal abscess drainage (1 patient), and small bowel obstruction (1 patient). Late surgical complications occurred in 23 patients (47\%). These consisted of wound infection ( $\mathrm{n}=23)$, wound/fascial dehiscence $(n=12)$, bowel obstruction/ileus $(n=5)$, intra-abdominal abscess $(\mathrm{n}=2)$, and anastomotic leak $(\mathrm{n}=1)$. Five patients experienced both post-surgical and late complications, indicating the development of a complication that persisted from the immediate post-surgical period to beyond the initial hospital discharge. 


\section{Treatment Response}

Nine patients achieved path CR $(17 \%, 90 \%$ confidence interval: [9\% - 27\%]). Eleven patients (20\%) were ypT0. Fourteen patients (26\%) and 29 patients (54\%) were ypT0-T1 and ypT0-T2, respectively. Thirty-two patients (59\%) experienced pathologic primary tumor downstaging. Thirty-two patients (59\%) and 42 patients (78\%) were ypN0 and ypN0-N1, respectively. Of the 32 patients with clinical nodal involvement who underwent surgery, 19 (59\%) were ypN0 and $21(66 \%)$ experienced pathologic nodal downstaging. Positive surgical margins occurred in 6 of the 49 patients who underwent surgical resection (12\%).

With a median follow-up period for alive patients of 17 months (range $2-54$ months), there have been 2 recurrence events. One patient recurred in the liver 11 months after initial surgery, subsequently underwent hepatic metastectomy, and is still alive. One patient recurred in the pelvic lymph nodes 17 months after initial surgery and is still alive. Overall survival and relapse free survival data have not yet matured and will be analyzed at a later date.

\section{DISCUSSION}

In this prospective multi-institutional phase II trial, neoadjuvant therapy consisting of capecitabine, oxaliplatin, and bevacizumab with RT was administered prior to curative surgical resection. This was to be followed by adjuvant chemotherapy consisting of 5-FU, leucovorin, oxaliplatin, and bevacizumab. The addition of oxaliplatin and bevacizumab to standard neoadjuvant CRT did not achieve the expected pathologic complete response rate of 30\%. Neoadjuvant therapy intensification demonstrated significant acute toxicity with 2 preoperative deaths ( 1 of which was attributed to study therapy), 53\% of patients with worst grade 3 toxicity, and $15 \%$ of patients with worst grade 4 toxicity. Late surgical complications were also common, with $47 \%$ of patients who underwent surgery experiencing some form of surgical complication, most commonly wound infection and wound/fascial dehiscence. Furthermore, 50\% of all patients began adjuvant chemotherapy, with only $30 \%$ receiving all treatment as per protocol.

This trial was designed and initiated prior to the release of results from several phase III studies investigating the addition of oxaliplatin to fluoropyrimide-based neoadjuvant CRT for locally advanced rectal cancer. The results were recently reported for NSABP R-04, a 4 arm trial comparing CI 5-FU-based CRT with or without oxaliplatin and capecitabine-based CRT with or without oxaliplatin for clinical stage II and III rectal cancer ${ }^{5}$. There were no differences in outcome between the oxaliplatin and no oxaliplatin arms, with pathologic CR rates of $21 \%$ and $19 \%$, respectively $(\mathrm{p}=0.46)$. However, there was a significant increase in grade $3 / 4$ diarrhea with the addition of oxaliplatin, with rates of $15 \%$ and $7 \%$, respectively (p $<0.001$ ). In another phase III trial, Gerard et al. examined capecitabine-based CRT to $45 \mathrm{~Gy}$ (Cape45) versus capecitabine and oxaliplatin-based CRT to $50 \mathrm{~Gy}$ (CapeOx50) ${ }^{8}$. There was no difference in the primary outcome of pathologic complete response, with path CR rates of $14 \%$ and $19 \%$, respectively $(\mathrm{p}=0.09)$. However, the rate of grade $3 / 4$ toxicity was significantly increased in the CapeOx50 arm (25\% vs. $11 \%$, p<0.001). Furthermore, Aschele et al. reported the results of the STAR-01 trial, a comparison of CI 5-FU CRT and the same regimen with weekly oxaliplatin ${ }^{16}$. Again, the addition of oxaliplatin significantly increased toxicity (grade $3 / 4$ toxicity $8 \%$ vs. $24 \%$, p $<0.001$ ) without affecting local tumor response. The preponderance of evidence supports the conclusion that the addition of oxaliplatin to fluoropyrimadine-based CRT increases toxicity without a benefit in local tumor response or path CR rates.

Bevacizumab has been associated with increased post-operative complication risk after major surgery when added to neoadjuvant CRT for several disease sites ${ }^{17-19}$. Willet at al. 
reported on a phase II trial examining the addition of bevacizumab to standard CI 5-FUbased CRT for locally advanced rectal cancer ${ }^{13}$. Five of 32 patients (16\%) experienced path CR and the 5 year actuarial local control and overall survival rates were both $100 \%$. Surgery occurred 7 - 10 weeks after the completion of neoadjuvant therapy, and the regimen containing bevacizumab appeared to be tolerable and safe. Postoperative complications included anastomotic leak with presacral abscess requiring drainage $(n=1)$, vaginal tear with presacral hematoma and abscess requiring drainage $(n=1)$, pelvic hematoma $(n=1)$, delayed healing of perineal incision $(n=2)$, ileus $(n=2)$, neurogenic bladder $(n=1)$, perforated ileostomy-stent related $(n=1)$, pulmonary embolus $(n=1)$, and wound infection $(n=3)$. A similar trial was performed by Crane et al., where patients with stage II or III rectal cancer were treated with neoadjuvant bevacizumab, capecitabine, and RT ${ }^{14}$. Eight of 25 patients (32\%) experienced path CR, with a 2 year actuarial local recurrence rate of $6 \%$. There were 3 major wound complications (12\%) that required surgical intervention: coloanal anastomotic dehiscence requiring completion APR $(n=1)$ and perineal wound dehiscences after initial APR $(\mathrm{n}=2)$. There were also five minor complications $(20 \%)$ that were managed without surgical intervention yielding a total surgical complication rate of $32 \%$. However, complication rate did differ by type of surgery, with 4 of 6 patients $(67 \%)$ who underwent APR developing a complication versus 2 of 10 patients (20\%) who underwent LAR. There was no detectable association between the time from last bevacizumab dose to surgery and surgical complication rate. Neither trial was able to specifically attribute the observed surgical complications to the addition of bevacizumab, with other possible explanations including patient selection, differences in surgical technique, and chance alone.

Dipetrillo et al. published a single institution trial of neoadjuvant bevacizumab and oxaliplatin with fluoropyrimide-based CRT for stage II - III rectal cancer ${ }^{20}$. Patients on this trial received 1 month of induction bevacizumab with modified infusional fluorouracil, leucovorin, and oxaliplatin (FOLFOX) 6 regimen followed by concurrent bevacizumab, oxaliplatin, CI 5-FU, and RT. The oxaliplatin dose had to be reduced from $50 \mathrm{mg} / \mathrm{m} 2$ to 40 $\mathrm{mg} / \mathrm{m} 2$ weekly due to gastrointestinal toxicity. The trial was terminated early after 26 of a planned 29 patients were enrolled due to excessive toxicity. Five of 25 patients (20\%) achieved a pathologic complete response. Grade 3/4 toxicity during neoadjuvant CRT occurred in 19 of 25 patients (76\%), consisting primarily of diarrhea $(n=16)$, pain $(n=11)$, and neutropenia $(\mathrm{n}=6)$. Nine patients $(36 \%)$ developed postoperative wound complications including infection $(\mathrm{n}=4)$, delayed healing $(\mathrm{n}=3)$, leak/abscess $(\mathrm{n}=2)$, sterile fluid collection $(\mathrm{n}=2)$, ischemic colonic reservoir $(\mathrm{n}=1)$, and fistula $(\mathrm{n}=1) .2$ patients $(12 \%)$ developed deep venous thrombosis. Only $25 \%$ of all patients were able to complete all treatment as per protocol. These results are similar to this trial with $67 \%$ of patients experiencing grade $3 / 4$ acute toxicity, only $30 \%$ of patients completing all therapy as per protocol, and a path CR rate of $17 \%$. It is important to note that the trials assessing the addition of oxaliplatin and/or bevacizumab to neoadjuvant chemoradiation have typically only reported tumor downstaging rates and toxicity to date. The more important endpoints of overall survival, relapse free survival, local recurrence, and distant recurrence are yet immature for most trials and are pending longer follow-up periods. The validity of path CR as a surrogate for long term oncologic outcome has not been firmly established in this disease site and conflicting reports as to the prognostic significance of path CR exist in the literature ${ }^{21-23}$. Path CR rates reflect response to neoadjuvant therapy only; however long term outcome may also be influenced by the intensification of adjuvant therapy in trials such as this.

Nine (18\%) and 23 (47\%) of the 49 patients who underwent surgery in this trial experienced post-surgical and late surgical complications, respectively. Five patients experienced both post-surgical and late complications, indicating the development of a complication that persisted from the immediate post-surgical period to beyond the initial hospital discharge. 
This event rate is slightly higher than the combined surgical complication rate of $48 \%$ reported by Dipetrillo et al.; however, there are 3 important differences between these 2 studies. (1) This phase II study is a multi-institutional trial with 11 participating centers compared with a single institution experience. (2) The concurrent chemotherapy regimen differed between trials, with capecitabine instead of 5-FU and all patients receiving $50 \mathrm{mg} /$ $\mathrm{m} 2$ oxaliplatin weekly in this trial. (3) The median time interval from last bevacizumab to surgery was shorter in this trial (median 7.6 weeks versus 8.9 weeks). The interval from the last dose of bevacizumab to surgery has been proposed as a possible risk factor for wound healing complications.

In summary, the primary endpoint of this trial was not reached, although the majority of patients experienced pathologic downstaging with the addition of bevacizumab and oxaliplatin to capecitabine and RT for locally advanced rectal cancer. Increased wound healing delays and complications may have been related to the addition of bevacizumab, oxaliplatin, or both. However, we were not able to attribute toxicity to a specific agent with certainty. Relapse free survival and overall survival data have not yet matured and will be reported in a future publication. Continued observation of these patients will establish the long term morbidity and efficacy of this combined modality approach.

\section{Acknowledgments}

This study was conducted by the Eastern Cooperative Oncology Group (Robert L. Comis, M.D.) and supported in part by Public Health Service Grants CA23318, CA66636, CA21115, CA27525, CA15488, CA49957, CA17145 and from the National Cancer Institute, National Institutes of Health and the Department of Health and Human Services.

\section{REFERENCES}

1. Siegel R, Ward E, Brawley O, Jemal A. Cancer statistics, 2011: the impact of eliminating socioeconomic and racial disparities on premature cancer deaths. CA Cancer J Clin. 2011; 61(4): 212-36. [PubMed: 21685461]

2. Gastrointestinal Tumor Study Group. Prolongation of the disease-free interval in surgically treated rectal carcinoma. N Engl J Med. 1985; 312(23):1465-72. [PubMed: 2859523]

3. Edge, SB.; American Joint Committee on Cancer. AJCC cancer staging manual. 7th ed.. Springer; New York: 2010.

4. Sauer R, Becker H, Hohenberger W, et al. Preoperative versus postoperative chemoradiotherapy for rectal cancer. N Engl J Med. 2004; 351(17):1731-40. [PubMed: 15496622]

5. Roh MS, Yothers GA, O'Connell MJ, et al. The impact of capecitabine and oxaliplatin in the preoperative multimodality treatment in patients with carcinoma of the rectum: NSABP R-04. J Clin Oncol. 2011; 29(suppl) abstr 3503.

6. Hess S, Blackstock AW. In vitro and in vivo evidence of its radiation sensitizing activity: Preclinical observation relevant to ongoing clinical trials [Abstract]. Proc 91st Ann Meeting Am Assoc Cancer Res (AACR). 2000:335. Abstract.

7. Raymond E, Faivre S, Chaney S, Woynarowski J, Cvitkovic E. Cellular and molecular pharmacology of oxaliplatin. Mol Cancer Ther. 2002; 1(3):227-35. [PubMed: 12467217]

8. Gerard JP, Azria D, Gourgou-Bourgade S, et al. Comparison of two neoadjuvant chemoradiotherapy regimens for locally advanced rectal cancer: results of the phase III trial ACCORD 12/0405-Prodige 2. Journal of clinical oncology : official journal of the American Society of Clinical Oncology. 2010; 28(10):1638-44. [PubMed: 20194850]

9. Hurwitz H, Fehrenbacher L, Novotny W, et al. Bevacizumab plus irinotecan, fluorouracil, and leucovorin for metastatic colorectal cancer. N Engl J Med. 2004; 350(23):2335-42. [PubMed: 15175435] 
10. Gorski DH, Beckett MA, Jaskowiak NT, et al. Blockage of the vascular endothelial growth factor stress response increases the antitumor effects of ionizing radiation. Cancer Res. 1999; 59(14): 3374-8. [PubMed: 10416597]

11. Lee CG, Heijn M, di Tomaso E, et al. Anti-Vascular endothelial growth factor treatment augments tumor radiation response under normoxic or hypoxic conditions. Cancer Res. 2000; 60(19):556570. [PubMed: 11034104]

12. Czito BG, Bendell JC, Willett CG, et al. Bevacizumab, oxaliplatin, and capecitabine with radiation therapy in rectal cancer: Phase I trial results. Int J Radiat Oncol Biol Phys. 2007; 68(2):472-8. [PubMed: 17498568]

13. Willett CG, Duda DG, di Tomaso E, et al. Efficacy, safety, and biomarkers of neoadjuvant bevacizumab, radiation therapy, and fluorouracil in rectal cancer: a multidisciplinary phase II study. Journal of clinical oncology : official journal of the American Society of Clinical Oncology. 2009; 27(18):3020-6. [PubMed: 19470921]

14. Crane $\mathrm{CH}$, Eng C, Feig BW, et al. Phase II trial of neoadjuvant bevacizumab, capecitabine, and radiotherapy for locally advanced rectal cancer. Int J Radiat Oncol Biol Phys. 2010; 76(3):824-30. [PubMed: 19464823]

15. Landry JC, Catalano P, Cohen SJ, et al. Phase II Study of Preoperative Radiation with Concurrent Capecitabine, Oxaliplatin and Bevacizumab followed by Surgery and Postoperative 5-FU, Leucovorin, Oxaliplatin (FOLFOX) and Bevacizumab in Patients with Locally Advanced Rectal Cancer: ECOG 3204. Int J Radiat Oncol Biol Phys. 2009; 75(3):S27-S28.

16. Aschele C, Pinto C, Cordio S, et al. Preoperative fluorouracil (FU)-based chemoradiation with and without weekly oxaliplatin in locally advanced rectal cancer: Pathologic response analysis of the Studio Terapia Adiuvante Retto (STAR)-01 randomized phase III trial. J Clin Oncol. 2009; 27(18s)(suppl) abstr CRA4008.

17. Scappaticci FA, Fehrenbacher L, Cartwright T, et al. Surgical wound healing complications in metastatic colorectal cancer patients treated with bevacizumab. J Surg Oncol. 2005; 91(3):173-80. [PubMed: 16118771]

18. Varadhachary GR, Wolff RA, Crane CH. Preoperative gemcitabine (gem) and bevacizumab (bev)based chemoradiation for resectable pancreatic adenocarcinoma. J Clin Oncol. May 20.2008 26(Supplement):4630.

19. Bege T, Lelong B, Viret F, et al. Bevacizumab-related surgical site complication despite primary tumor resection in colorectal cancer patients. Ann Surg Oncol. 2009; 16(4):856-60. [PubMed: 19156464]

20. Dipetrillo T, Pricolo V, Lagares-Garcia J, et al. Neoadjuvant bevacizumab, oxaliplatin, 5fluorouracil, and radiation for rectal cancer. Int J Radiat Oncol Biol Phys. 2012; 82(1):124-9. [PubMed: 20947267]

21. García-Aguilar J, Hernandez de Anda E, Sirivongs P, Lee S-H, Madoff R, Rothenberger D. A Pathologic Complete Response to Preoperative Chemoradiation Is Associated With Lower Local Recurrence and Improved Survival in Rectal Cancer Patients Treated by Mesorectal Excision. Diseases of the Colon \& Rectum. 2003; 46(3):298-304. [PubMed: 12626903]

22. Pucciarelli S, Toppan P, Friso ML, et al. Complete Pathologic Response Following Preoperative Chemoradiation Therapy for Middle to Lower Rectal Cancer Is Not a Prognostic Factor for a Better Outcome. Diseases of the Colon \& Rectum. 2004; 47(11):1798-807. [PubMed: 15622571]

23. Park IJ, You YN, Agarwal A, et al. Neoadjuvant treatment response as an early response indicator for patients with rectal cancer. Journal of clinical oncology : official journal of the American Society of Clinical Oncology. 2012; 30(15):1770-6. [PubMed: 22493423] 


\section{Condensed abstract}

The addition of oxaliplatin and bevacizumab to fluoropyrimidine-based neoadjuvant chemoradiation for locally advanced rectal cancer resulted in pathologic tumor downstaging in the majority of patients, with a pathologic complete response rate of $17 \%$. Increased wound healing delays and complications may have been related to the addition of bevacizumab, oxaliplatin, or both. 


\section{Table 1}

Patient Characteristics.

\begin{tabular}{lll} 
Characteristic & No. & \% \\
\hline Eligible patients & 54 & $(100)$ \\
Gender & & \\
Male & 37 & $(69)$ \\
Female & 17 & $(32)$ \\
Race & & \\
White & 37 & $(69)$ \\
Black & 12 & $(22)$ \\
Hispanic & 2 & $(4)$ \\
Other & 3 & $(6)$
\end{tabular}

Performance status

$\begin{array}{lll}0 & 42 & (78) \\ 1 & 12 & (22)\end{array}$

Clinical T stage

$\begin{array}{lll}3 & 50 & (93) \\ 4 & 4 & (7)\end{array}$

Clinical N stage

$\begin{array}{lll}0 & 17 & (32) \\ 1 & 30 & (56) \\ 2 & 5 & (9) \\ X & 2 & (4)\end{array}$

Surgical procedure

$\begin{array}{lll}\text { Surgery } & 49 & (91) \\ \text { TME } & 37 & (69) \\ \text { APR } & 11 & (20) \\ \text { LAR } & 16 & (30) \\ \text { LAR/coloanal anastomosis } & 22 & (41) \\ \text { Other } & 1 & (2) \\ \text { Unknown } & 1 & (2) \\ \text { Median age } & 54 \text { years (range 26-83) }\end{array}$

$\mathrm{TME}=$ total mesorectal excision

$\mathrm{APR}=$ abdominoperineal resection

$\mathrm{LAR}=$ low anterior resection 


\section{Table 2}

Highest hematologic toxicity for each patient during chemoradiation $(\mathrm{n}=55)$.

\begin{tabular}{llll}
\hline Characteristic & Grade 2 & Grade 3 & Grade 4 \\
\hline Anemia & 6 & 3 & 2 \\
Leukopenia & 10 & 7 & 1 \\
Lymphopenia & 3 & 7 & 1 \\
Neutropenia & 4 & 9 & 1 \\
Thrombocytopenia & 2 & 3 & - \\
\hline Worst degree & 8 & 16 & 4 \\
\hline
\end{tabular}




\section{Table 3}

Highest non-hematologic toxicity for each patient during chemoradiation $(\mathrm{n}=55)$.

\begin{tabular}{lllll}
\hline Characteristic & Grade 2 & Grade 3 & Grade 4 & Grade 5 \\
\hline Hypotension & 4 & - & - & - \\
Fatigue & 12 & 7 & 1 & - \\
Weight loss & 2 & 2 & - & - \\
Rash/desquamation & - & 2 & - & - \\
Anorexia & 6 & 2 & - & - \\
Dehydration & 4 & 5 & - & - \\
Diarrhea & 12 & 6 & 1 & - \\
Aspiration & - & - & - & 1 \\
Nausea & 10 & 2 & 1 & - \\
Proctitis & 3 & 1 & - & - \\
Vomiting & 5 & 3 & - & - \\
CNS hemorrhage & - & - & 1 & - \\
Sensory neuropathy & 2 & 4 & - & - \\
Abdominal pain & 3 & 3 & 1 & - \\
Rectal pain & 5 & 7 & 2 & - \\
Deep vein thrombosis & - & - & 2 & - \\
Death NOS & - & - & - & 1 \\
\hline Worst degree & 18 & 23 & 5 & 2 \\
\hline NOS = not otherwise specified & & & \\
\hline
\end{tabular}

